\title{
COMPARISON OF EFFICACY BETWEEN THE PED-ANAM AND ANAM INSTRUMENT AND THE WECHSLER INTELLIGENCE SCALES IN NEUROPSYCHIATRIC LUPUS
}

Jaqueline Cristina de Amorim (Faculdade de Ciências Médicas - UNICAMP , Campinas, SP, Brasil), Flávia Allegretti Cavaletti (Faculdade de Ciências Médicas - UNICAMP , Campinas, SP, Brasil), Paula Teixeira Fernandes (Faculdade de Educação Física - UNICAMP, Campinas, SP, Brasil), Simone Thiemi Kishimoto (Faculdade de Ciências Médicas - UNICAMP , Campinas, SP, Brasil), Roberto Marini (Faculdade de Ciências Médicas - UNICAMP , Campinas, SP, Brasil), Lilian Tereza Lavras Costallat (Faculdade de Ciências Médicas - UNICAMP , Campinas, SP, Brasil), Simone Appenzeller (Faculdade de Ciências Médicas - UNICAMP , Campinas, SP, Brasil)

\section{BACKGROUND}

Among the neuropsychiatric manifestations of Systemic Lupus Erythematosus, cognitive losses are frequent. The translation and validation of Ped-Anam (Automated Pediatric Neuropsychological Assessment) for Children or Anam (Automated Neuropsychological Measurement) for adults allows the screening of these functions. Therefore, the objective of this study was to compare the results of this automated battery with the Wechsler Intelligence Scales.

\section{MATERIALS AND METHODS}

This is a cross-sectional quantitative study. PedAnam has been translated into Portuguese following the "Guidelines for the process of transcultural adaptation of self-report measures". The battery is composed of 10 subtests that were related according to area, content and evaluation to the subtests of the Wechsler scales. Wechsler Intelligence Scale for Children (WISC) and Wechsler Adult Intelligence Scale (WAIS) were applied to children. After the application, the results were compared.

\section{RESULTS}

32 adults with a mean of 27 years of SD \pm 11.3 and 12 children with an average of 12 years of SD \pm 3.8 were included. 31 women and 13 men. The subjects responded to the two tests on different days due to the time of the applications. The results were analyzed using the Spearman test, considering the closer to 1 the greater the positive correlation and the closer to -1 the negative correlation. The Code SubstitutionLearning sub-test is more similar when compared to the digit span $(r=0.958)$, both assess the speed of perceptual comparison. Next, Mathematical Processing with Arithmetic Subtest $(r=0.640)$, measurement of basic computational skills, concentration and mathematical aspects. Sub-tests related to memory can be observed moderate correlation $(r=0.413)$. While the Matching Grids and Simple Reaction Time subtests are moderately related Digit Symbol Coding. Finally, subtests related to executive functions Spatial Processing with Block Design $(r=0.286)$.

\section{CONCLUSION}

The Wechsler scales are larger when compared to computerized anam batteries. The computerized battery presented correspondence with the content and the result varied from reasonable to excellent. Is a good battery for tracking cognitive demands, exploring different areas of cognition. The translation and initial validation suggest that the battery can be a useful screening tool. Further research is needed to confirm the excellent accuracy in identifying cognitive impairment as well as its usefulness in detecting clinically relevant changes in cognition over time. 